1. McMahon, H.T. \& Mills, I.G.Curr. Opin Cell Bial. 16, 379-391 (2004)

2. Lee, M. C S etal. Cell 122,605-617(2005)

3 Matsuoka, K.et al. Cell 93, 263-275 (1998)

4. Hristova, K.et al. J. Mol. Bial. 290, 99-117 (1999)

\section{SOLID-STATE PHYSICS}

\section{Spin in the slow lane}

\author{
Bart van Wees
}

\section{Electrons were until recently thought to transport their charge and spin equally freely through metals and semiconductors. Now it seems that spin can lag considerably behind charge.}

The recognition that electrons can transport through a solid not just charge, but also spin (the intrinsic 'rotation' of electrons, which gives rise to their magnetic moment), sowed the now burgeoning field of spintronics. As charge and spin are properties of individual electrons, it is tempting to conclude that both will be transported equally efficiently. But Weber et al. ${ }^{1}$, writing on page 1330 of this issue, show that the diffusion of electron spin can be substantially slower than that of electron charge. This could have important implications for spin-based electronics.

The ability to generate, study and use phenomena such as spin injection, spin currents and spin accumulation, in metals as well as semiconductors, has made it imperative to discover how similar — or different - spin and charge transport are. In metals, no substantial differences have been observed ${ }^{2}$; but in semiconductors, experiments in the past decade have already indicated that charge and spin diffusion act differently ${ }^{3}$. The comparison of the two transport mechanisms is simplified by assuming that the electron spin (or its associated magnetic moment) can point only up or down along a specific axis. In conventional charge currents, these spin-up and spin-down electrons move in the same direction. But when, in the absence of a charge current, spinup electrons move in one direction, and an equal number of spin-down electrons move in the opposite direction, a spin current is established, with magnetic moment effectively being transported in one direction.

Usually, electron motion is diffusive: scattering on impurities, lattice defects and lattice vibrations will, after some time, randomize the direction of an electron's movement. The more scattering that takes place, the slower the electron diffusion will be. As this effect applies equally to spin-up and spin-down electrons, the naive expectation is that the effective rates of charge and spin diffusion should be the same. But this neglects the electrostatic repulsion, or Coulomb interaction, that exists between electrons. The Coulomb interaction does not affect the diffusion of charge, as it does not change the total momentum of the

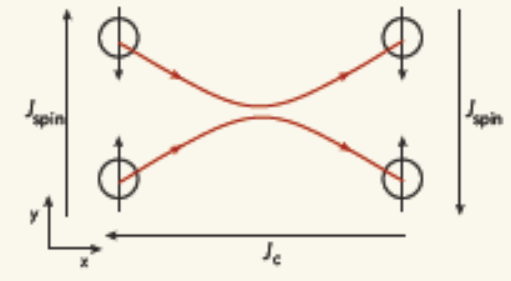

Figure 1|Spin drag. Two electrons are moving in the $x$ - $y$ plane with opposite directions of spin. In this particular case, they initially contribute to a charge current $J_{c}$ in the negative $x$-direction (conventionally, electric current is depicted as a flow of positive charges, in the opposite direction to the actual electron flow). Because the spin-tip electron is moving upwards and the spin-down electron downwards, there is also a net spin-up current $J_{\text {qin }}$ in the positive $y$-direction. Because of Coulomb repulsion as the electrons near each other, and the resulting exchange of momentum, their direction of motion in the $y$-direction, and so their contribution to the spin current, is reversed. Their contribution to the charge current, however, is unaffected. Thus, unlike the diffusion of charge, the diffusion of spin is slowed down - the spin Coulomb drag demonstrated by Weber et $a L^{1}$. (Modified from ref. 1.)

electrons. But it does reduce the diffusion rate of spin through scattering between spin-up and spin-down electrons (Fig. 1) - the effect known as spin Coulomb drag that has now been demonstrated experimentally by Weber and colleagues ${ }^{1}$.

Measuring the relevant experimental parameter, the spin diffusion constant $D$, necessitated a clever technique based on a so-called spin grating ${ }^{4}$. The authors first used ${ }^{1}$ the interference between two laser beams polarized linearly, but at right angles to each other, to 'pump' their sample - a crystal of the semiconductor gallium arsenide - with a very short light pulse. The two beams met in the plane of the semiconductor at an angle, so their relative phase changed linearly along its surface. This induces a periodic variation in spin density - the spin grating - in the quantum wells in which the electrons are confined. Here, regions of excess spin-up and excess spin-down electrons alternate with a spatial period $L$ that is dependent on the angle of incidence of the two beams. This spin grating decays with time, partially through intrinsic relaxation of the spin, and partially as a result of diffusion between the excess spin-up and spin-down regions (on a typical timescale $T=L^{2} / D_{s}$ given by the laws of diffusion).

The authors then applied a further linearly polarized, 'probe' laser pulse to their crystal. This brought the Faraday effect into play, which dictates that the interaction of a beam of light with a magnetic field - in this case, that induced by the electron spins - will bring about a (slight) rotation of the polarization of the reflected beam. The angle of rotation depends on the induced magnetization (and thus the spin density), so the decay of the spin grating can be investigated by varying the delay between the pump and probe pulses. By ascertaining the effective decay time as a function of the spatial period $L$, the authors determined the relative importance of diffusion and relaxation, and obtained values for both $D_{\mathrm{s}}$ and the intrinsic spin relaxation time. They found that the rate of spin diffusion indicated by $D_{s}$ was considerably slower than the charge diffusion rates obtained from conventional electronic measurements.

In theoretical calculations ${ }^{5,6}$, spin Coulomb drag is expressed by a parameter known as the spin-drag transresistivity; this relates the current in each of the spin channels (spin up or spin down) to the effective electric field in the opposite spin channel. The significance of the transresistivity depends on the number of spatial dimensions considered, the strength of the various interactions between the electrons, and the conventional electronic resistivity. If this last quantity is small, as it is in good conductors such as metals, the effect of spin drag will be relatively small ${ }^{2}$. In the two-dimensional system used by Weber et al. ${ }^{1}$, however, the effect of spin Coulomb drag turns out to be considerable. Reducing further to a one-dimensional system has revealed that spin and charge can even separate ${ }^{7}$, with the two intrinsic electron properties developing their own distinctive transport modes. These findings are bound to stimulate further research. What is already abundantly clear, however, is that spin and charge both move at their own pace.

Bart van Wees is in the Department of Applied

Physics and the Materials Science Centre, Groningen University, Nijenborgh 4.13, 9747 AG Groningen, The Netherlands. e-mail:b.j.van.wees@rug.nl

1. Weber, C.P. etal Nature 437,1330-1333(2005).

2. Jedema, F. L, Heersche, H. B, Filip, A. T, Baselmans, I I A. \&van Wees, B. I Nature 416, $713-716$ (2002).

3. Kikkawa, I M. \& Awschalom, D.D. Nature 397, 139-141 (1999)

4. Cameron, A.R, Riblet, P.\& Millec, A.Phys. Rex Lett. 76, 4793-4796(1996).

5. DAmica L \& Vignale, G. Phys Rex B68,045307 (2003)

6. Flensberg, $K_{\gamma}$ Jensen, T. S.\&. Mortensen, N.A.Phys Rev. B 64, 245308 (2007).

7. Auslaender, O.M.etal. Science 308, 88-92 (2005). 